\title{
Baden wie Burkhard III. von Hallwyl im 16. Jahrhundert
}

\author{
Martina Föhn \\ Buttwil, Schweiz
}

\section{Schlüsselwörter}

Balneotherapie · Bader · Mittelalterliche Rezepturen · Heilkräuter · Schweiz

\section{Zusammenfassung}

Burkhard III. von Hallwyl schrieb um das Jahr 1580 ein Arzneibuch mit 2000-3000 Rezepturen. Mit diesem Werk in frühneuhochdeutscher Sprache wollte er lesekundige Personen ansprechen, die keine Lateinkenntnisse besassen. Viele seiner niedergeschriebenen Rezepturen beschäftigen sich mit dem Thema «Baden». Dieses Essay beschreibt die Geschichte des Badens in Mitteleuropa mit der Entstehung der Badestuben und dem Rückgang des Badewesens im Mittelalter. Zwei Baderezepturen aus dem Buch Burkhards werden analysiert: Rezeptur 1:«Volgendt guote underricht wie man das baad sol machen gar für mancherleY gepresten»; Rezeptur 2: «Ein guot baad zuo den erkalteten glideren». Rezeptur 1 wird bei Indikationen wie rheumatischen Erkrankungen, Hauterkrankungen, Wunden, Schmerzen, Steifheit, aber auch Lähmungen, Gliederschwund, Frauenkrankheiten und Schlag eingesetzt. Rezeptur 2 ist insbesondere bei rheumatischen Erkrankungen, Gicht, Schmerzen und Steifheit der Glieder indiziert. Mithilfe botanischer, historischer, medizinisch-therapeutischer und zum Teil sprachwissenschaftlicher Literatur erfolgte die Analyse der Rezepturen. Viele der verwendeten Heilpflanzen und Stoffe konnten eindeutig identifiziert werden.

(c) 2015 S. Karger GmbH, Freiburg

\section{Einleitung}

Für die Ethnobotanik und Ethnomedizin sind Arzneimittelrezepturen früherer Epochen von grosser Bedeutung. Sie geben Auskunft darüber, welche Heilpflanzen verwendet worden sind und in welchen Pflanzen allenfalls

Überarbeitete und aktualisierte Version einer Projektarbeit im Rahmen des CAS «Ethnobotanik und Ethnomedizin» an der Universität Zürich, Schweiz.

\section{Keywords}

Balneotherapy · Barber surgeon · Medieval formulas · Medicinal herbs . Switzerland

\section{Summary}

Herbal Formulas for Bathing in the 16th Century

Burkhard III. von Hallwyl (Switzerland) was the author of a herbal written in 1580. This herbal contains 2000-3000 formulas. The author's intention of this herbal was a compendium for laypeople without knowledge in Latin or a higher education. Many of the formulas deal with 'bathing'. Therefore, this essay focusses on balneotherapy and the development of bathing culture in Central Europe from the first medieval bathhouses to the cultural collapse in the 16th century. Two formulas for bathing essences were analyzed: Formula 1: 'Volgendt guote underricht wie man das baad sol machen gar für mancherleY gepresten' (good practice how you should prepare a bath for many afflictions); Formula 2: 'Ein guot baad zuo den erkalteten glideren' (a good bath for cold limbs). Formula 1 is indicated for rheumatic pain, skin diseases, wounds, pain, stiffness, paralysis, cancer, gynecological diseases, and stroke. Formula 2 is specifically indicated for rheumatic pain, gout, pain, and stiffness. Our analysis is based on a comparison with botanical, medical-historical, and linguistic literature. Many of the formulas' ingredients could be clearly identified.

(c) 2015 S. Karger $\mathrm{GmbH}$, Freiburg

ein Potenzial für die moderne Medizin steckt. So sind einige Kräuter, die Burkhard III. von Hallwyl (Schweiz) in seinem Arzneibuch von 1580 verwendete, heute noch gebräuchlich (z.B. Salvia officinalis). Andere hingegen sind in Vergessenheit geraten oder wegen eines fehlenden Wirksamkeitsnachweises nicht mehr in Verwendung (z.B. Stachys officinalis). Ein beachtlicher Teil der Rezepturen in Burkhards Arzneibuch beschäftigt sich mit der Zubereitung und Anwendung von Bädern. Die Balneotherapie erlebte zur Zeit Burkhards im 16. Jahrhundert einen Höhepunkt.

\section{KARGER}

Fax +497614520714 Information@Karger.com www.karger.com
1015-0684/15/0274-0219\$39.50/0
Martina Föhn

Galizistrasse 31, 5632 Buttwil, Schweiz

martina.foehn@bluewin.ch 


\section{Geschichte der Balneotherapie}

Seit der Antike ist die Badekultur ein Vergnügen der Menschheit [1]. So war das Baden bereits in frühgeschichtlicher Zeit im Orient beliebt. Im griechischen Kulturraum entwickelte sich das Badewesen nach dem Vorbild altorientalischer und indischer Kulturen. Die Erfindung der römischen Fussbodenheizunglöste die Kaltbäder durch die Warmbäder ab. Im 14. und 15. Jahrhundert nahm die Beliebtheit der Warmbäder in Mitteleuropa zu. Wannenbäder mit Kräuterzugaben galten als heilend [2]. Neben dem Reinigungsbad spielte das Schwitzbad eine zentrale Rolle. Diesem Bädertyp schrieb man eine vorbeugende und heilende Wirkung zu, z.B. bei Lepra. Beim Dampfbad wurde unter einer Sudblase, in der sich Wasser mit verschiedenen Heilkräutern befand, ein Feuer entzündet. Dampfbäder führten zum Schwitzen und zum Ausscheiden schlechter Körpersäfte. Kräuterbäder wurden gegen Geschwüre, Hautleiden und rheumatische Erkrankungen verabreicht.

Öffentliche Bäder gab es im 16. Jahrhundert zunächst nur dort, wo Klöster ihre Bäder der Bevölkerung zur Verfügung stellten. Erst allmählich entstanden öffentliche Badestuben. Aus diesem Bedürfnis heraus entwickelte sich der Beruf des «Baders». Der Bader schröpfte, liess zur Ader, bereitete Schwitz- und Wannenbäder vor, massierte und half als Wundarzt bei medizinischen Problemen. Mit der «Badenfahrt» bezeichnete man im 16. Jahrhundert die Reise zu Wildbädern mit mineralischen oder Thermalquellen, die man in der Hoffnung auf Heilung zu Badekuren aufsuchte. Die Körperreinigung spielte dabei oft nur eine sekundäre Rolle. An erster Stelle stand die Geselligkeit. Bereits im 13. und 14. Jahrhundert gab es Verordnungen, die das gemeinsame Baden von Männern und Frauen in der öffentlichen Badstube verboten. Freudenhaus und Badhaus waren nämlich oft dasselbe.

Mit der Entdeckung Amerikas handelte sich das alte Europa eine «neue» Krankheit ein: die Syphilis. Der Ausbruch dieser Krankheit bescherte dem Badehaus als Hauptinfektionsherd im 15. und 16. Jahrhundert ein jähes Ende. Verteuerung und Verknappung von Brennmaterial trugen zusätzlich zum Untergang dieser Einrichtung bei. Auch ständig aufflammende Pestepidemien sowie die Lepra blieben nicht ohne Wirkung auf das Badeleben. Mit dem Aufkommen von eigenen Badezimmern in der Mitte des 20. Jahrhunderts verloren die öffentlichen Badeanstalten ihren Reiz. Besonders in der jüngsten Zeit hat aber die Badekultur mit dem Aufkommen von «Wellness-Einrichtungen» wieder an Bedeutung gewonnen.

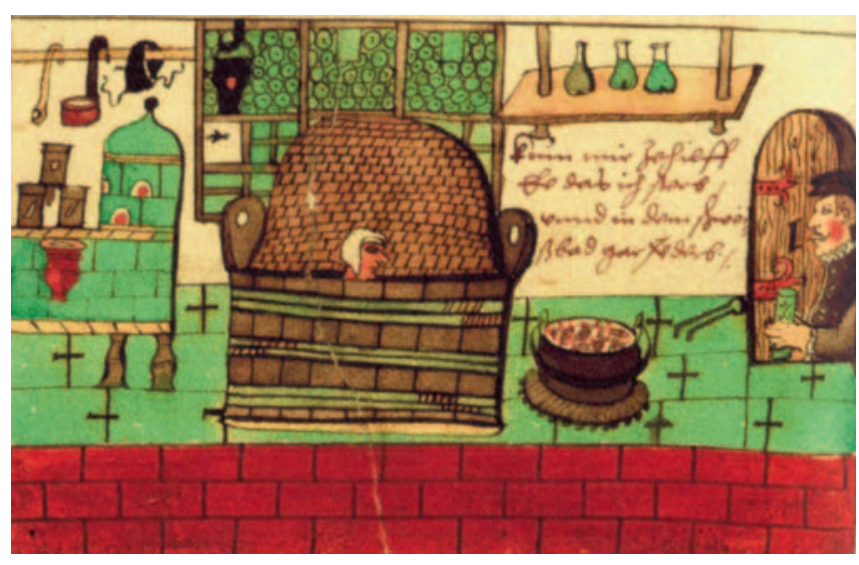

Abb. 1. Versuchslabor des Burkhard III. mit Bad (Abbildung aus dem Arzneibuch Burkhards III. im Staatsarchiv von Bern [3]).

\section{Arzneibuch des Burkhard III. von Hallwyl}

Obwohl Burkhard (1535-1589) über keine akademische Ausbildung verfügte, eignete er sich vertiefte Kenntnisse in der Arzneimittelherstellung an. In seinem Versuchslabor im Schloss Hallwyl entwickelte er mithilfe von Destillierapparaturen und einem Bad (Abb. 1) eigene Rezepturen.

Von Burkhards Arzneibuch existieren heute dreizehn Abschriften aus dem 17. Jahrhundert. In dieser Arbeit wurde die Transkription einer Abschrift aus dem Jahr 1611 verwendet. Sie stammt von Peter Gasser aus Kleinandelfingen und Frieda Schmid-Bai aus Oberägeri und wurde für das Heimatkundliche Archiv in Andelfingen angefertigt. Diese Transkription wurde mit dem Original des Arzneibuchs im Staatsarchiv in Bern und einer Abschrift in der Ausstellung «Forschen und Heilen» [2] im Museum im Schloss Hallwyl verglichen. Für die Untersuchung wurden zwei Baderezepturen ausgewählt:

\section{Rezeptur 1: "Volgendt guote underricht wie man das}

baad sol machen gar fÜr mancherleY gepresten»

Zum ersten nim diße nachgeschribnen kreÜtter, wurtzen und sammen: laffandel, wermuot, salbinen, bethonien, fischmÜntz, rott baadkrautt, rott bugcken, wohlgemut, empfen kraut, 1Ybstickel, neslen wurtzen, jedes ein handvol, demnach neßlen sammen 4 lodt, reckholterbere 4 vierling, diße st $U ̈ c k$ thuo alle in ein secklin und nim ein handvol schwebel und 4 haller lorberi darzuo, und jn einem großen keßel gesotten also vil, das des gesottnen wassers 2 bÜttenen voll wirt, jn der einten sol man 3 tag baden, jn der anderen auch so lang, und kein ander waßer sol gebrucht werden, dan deßgesottnen, und allwegen sol man nÜechter baden, wo man das erzeÜgen mag, und wil man das das baad nit stinckendt werd, so sol man darin thuon weißen augstein 4 lodt und weißen wierauch 2 lodt. 


\section{Rezeptur 2: «Ein guot baad zuo den erkalteten} glideren»

Nim salbinen, rautten, wŠrmuoth, braun bethonien, fischmÜntz, rotte bachmÜntz, rotten constantz, rott buggelen, špfen kraut, neßlen wurtz und sammen, lYbstickel, reckholter bere, jedes ein große hand voll, zuosammen gethan, und theils in 2 secklin, darnach nim 1 hand vol schwebel, 1 handt vol lorbonen, das so obstaht seÜd alles jn waßer das also ob den kreÜtteren gesotten seYe, mach 2 zÜber, und baad so lang du magst, nach dem eßen sitz in den anderen zÜber und baad auch wie vor, thuo das 3 tag nacheinanderen, demnach mach ein frisch warm baadt wie vor, und baad wie man sonst badet, diß baad ist beßer dan alle beder, wŠre dir das marck jn den beinen erfroren oder das bluot erkaldt oder sonst ander presten.

\section{Methoden für die Analyse der Baderezepturen}

Die beiden Rezepturen im Original und in der Abschrift von Hallwyl wurden zusammen mit der Transkription auf folgende Kriterien untersucht:

- Vorkommen der Glossen (Bezeichnung für Pflanzen in einer älteren Sprache, die heute nicht mehr verwendet werden) in der Schweiz,

- regionale Verwendung der Glossen,

- Vorkommen der Glossen im 16. Jahrhundert,

- verwandtschaftliche Bezeichnungen in anderen Sprachen,

- sprachwissenschaftliche Hinweise,

- mögliche sprachliche Abwandlungen,

- Häufigkeit der Nennung in verschiedenen Heilpflanzenbüchern,

- Samenfunde im und um das Schloss Hallwyl,

- natürliche Vorkommen in der Umgebung des Schlosses Hallwyl,

- mögliches Vorhandensein in der Umgebung des Schlosses Hallwyl durch früheren Import und Anpflanzung in Gärten,

- mögliche Anwendung der Pflanze äusserlich oder in einem Bad,

- Indikation der verwendeten Pflanze zur Zeit des Burkhard III.,

- Indikation der verwendeten Pflanze in der heutigen Zeit,

- charakteristische Eigenschaft und Element nach der Signaturenlehre,

- botanische Merkmale, wie z.B. Färbungen von Stängeln und Blütenfarben,

- verwendete Pflanzenteile,

- Sammelzeit der Pflanze.

Idealerweise erfüllen die gesuchten Pflanzen alle oder zumindest eine Vielzahl der geforderten Kriterien. Für die Analyse der Pflanzen wurden weiterhin Vergleiche zu an- deren Rezepturen aus dem Arzneibuch Burkhards oder aus weiteren Arzneibüchern angestellt.

Zur Analyse der Pflanzennamen wurden verschiedene Literaturquellen hinzugezogen:

- Heinrich Marzell: Wörterbuch der deutschen Pflanzennamen, Band 5, Register. Leipzig, S. Hirzel Verlagsbuchhandlung, fotomechanischer Nachdruck der Erstausgabe 1958. Lizenzausgabe. Köln, Parkland, 2000.

- Matthias von Lexer: Mittelhochdeutsches Taschenwörterbuch, ed 38. Stuttgart, S. Hirzel, 1992.

- Carl Jakob Durheim: Schweizerisches Pflanzen-Idiotikon. Bern, Verlag von Huber \& Comp. (Körber), 1983, unveränderter Neudruck von 1856.

- Johannes Arends: Volkstümliche Namen, ed 18. Berlin, Springer, 2005

- www.heilpflanzen-welt.de/buecher/Hahnemann-Apothekerlexikon/1.9.09.

- Rudolf Creutz: Das mittelalterliche medizinisch-botanische Vokabularium "Alphita» in Quellen und Studien zur Geschichte der Naturwissenschaften und der Medizin (P. Diepgen und J. Ruska), Band 7. Berlin, Springer, 1940.

- Georg Madaus: Lehrbuch der biologischen Heilmittel, Heilpflanzen, Band III. Leipzig, Thieme, 1938.

Die Indikation der Rezepturen resultierte aus der Übereinstimmung aller Indikationen der Pflanzen, die in der jeweiligen Rezeptur Burkhards verwendet worden waren. Ausserdem wurden die Mass- und Volumenangaben der verwendeten Materialien analysiert. Abschliessend wurden die beiden Rezepturen miteinander verglichen.

\section{Analyse der Rezepturbestandteile}

Die Pflanzen und Stoffe, die für die Rezepturen verwendet wurden, sind mit volkstümlichen Namen in frühneuhochdeutscher Sprache bezeichnet. Diese Glossen sind heute grösstenteils nicht mehr gebräuchlich, ihre sprachwissenschaftlichen Weiterentwicklungen jedoch schon noch. Die Glossen unterschieden sich innerhalb des deutschsprachigen Raums. Jedoch wurden auch innerhalb des schweizerdeutschen Sprachgebiets lokal verschiedene Dialekte gesprochen, die sich in der unterschiedlichen Namensgebung der Heilpflanzen äussern. In dieser Zeit gab es keine festen grammatikalischen Regeln. Die Schreibweise der Glossen in Original, Abschrift und Transkription variiert. Der Bildungsstand der Personen, die die Abschriften machten, ist nicht bekannt. Beim Abschreiben wurden Fehler gemacht, wie aus dem Weglassen oder in der Satzstellung von Wörtern ersichtlich ist. Oftmals wurde eine Glosse für mehrere Pflanzenarten benutzt, und umgekehrt führen verschiedene Glossen auf ein- und dieselbe Pflanzenart zurück. Tabelle 1 zeigt die 
Tab. 1. Resultate der analysierten Pflanzennamen (der transkribierte Name stammt aus der Transkription der Abschrift des Arzneibuchs des Burkhard III.)

\begin{tabular}{|c|c|c|c|}
\hline \multicolumn{2}{|l|}{ Rezeptur 1} & \multicolumn{2}{|l|}{ Rezeptur 2} \\
\hline Transkribierter Name & lateinischer bzw. deutscher Name & transkribierter Name & lateinischer bzw. deutscher Name \\
\hline \multirow[t]{2}{*}{ laffandel } & Lavandula angustifolia Miller & & \\
\hline & & rautte & Ruta graveolens L. \\
\hline wermuot & Artemisia absinthium L. & wŠrmuoth & Artemisia absinthium L. \\
\hline salbine & Salvia officinalis $\mathrm{L}$. & salbine & Salvia officinalis $\mathrm{L}$. \\
\hline bethonie & Stachys officinalis $\mathrm{L}$. & braune bethonie & Stachys officinalis L. \\
\hline fischmÜntz & Mentha aquatica L. & $\begin{array}{l}\text { fischmÜntz } \\
\text { rotte bachmÜntz }\end{array}$ & $\begin{array}{l}\text { Mentha aquatica L. } \\
\text { Mentha aquatica L. }\end{array}$ \\
\hline \multirow[t]{2}{*}{ rott baadkrautt } & Origanum vulgare L. & & \\
\hline & & rotte constantz & Origanum vulgare L. \\
\hline rott bugcken & Artemisia vulgaris $\mathrm{L}$. & rott buggelen & Artemisia vulgaris $\mathrm{L}$. \\
\hline wohlgemut & Origanum vulgare $\mathrm{L}$. & & \\
\hline empfen kraut & nicht sicher identifiziert & Špfen kraut & nicht sicher identifiziert \\
\hline lYbstickel & Levisticum officinale Koch & lYbstickel & Levisticum officinale Koch \\
\hline neslen wurtz & Urtica dioica $\mathrm{L}$. & neßlen wurtz & Urtica dioica $\mathrm{L}$. \\
\hline neßlen sammen & Urtica dioica L. & neßlen sammen & Urtica dioica L. \\
\hline reckholterbere & Juniperus communis L. s. 1 . & reckholter bere & Juniperus communis L. s. 1. \\
\hline schwebel & Schwefel & schwebel & Schwefel \\
\hline lorberi & Laurus nobilis L. & lorbonen & Laurus nobilis L. \\
\hline weißen augstein & Bernstein & & \\
\hline weißen wierauch & Weisser Weihrauch & & \\
\hline
\end{tabular}

Abb. 2. Blüte von Lavandula angustifolia (Foto: Martina Föhn).

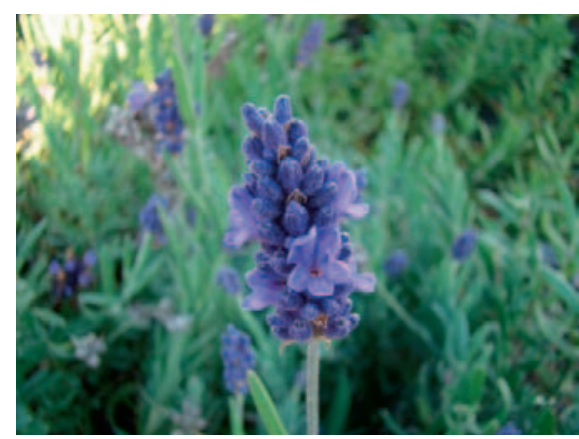

Zusammenfassung der analysierten Pflanzennamen in beiden Rezepturen.

Im Folgenden werden die in den Rezepturen verwendeten Heilpflanzen und Stoffe porträtiert.

Laffandel - (Rezeptur 1) - Lavandula angustifolia Miller - Echter Lavendel

Etymologie: Der mittellateinische Name «lavandula» kann vom Lateinischen «lavare» (= waschen) abgeleitet werden, weil man das aromatische Kraut gern dem Waschwasser oder den Bädern zusetzte.

Frühere Verwendung: Nach Bock [4] wirken Blüten und Kraut von «Lafander» gegen «Lame Glieder, Zittern, Schwindel, der von Kälte kommt». Seit dem 16. Jahrhundert wird Lavendel als beruhigendes, nervenstärkendes und krampflösendes Mittel verwendet.

Heutige Verwendung: Heute wird Lavendel (Abb. 2) als Spasmolytikum, Karminativum, Stomachikum und
Diuretikum sowie zur Wundbehandlung eingesetzt. Die Droge ist ebenso Bestandteil von Beruhigungsbädern und Kräuterkissen bei Schlafstörungen. Sie enthält ätherisches Öl mit den Hauptbestandteilen Linalylacetat und Linalool. Lavendelblütenextrakt wird bei Gillert und Rulffs [5] gegen Frostbeulen, chronisch kalte Füsse sowie bei allgemeinen Durchblutungsstörungen eingesetzt.

\section{Wermuot (Rezeptur 1) und WŠrmuoth (Rezeptur 2)}

- Artemisia absinthium L. - Wermut

Etymologie: Lexer [6] definiert «Wermuot» als Artemisia absinthium L. Häufig wird er mit «Wurm» in Verbindung gebracht, da die Pflanze früher gegen Eingeweidewürmer verwendet wurde. Andere Formen sind an «warm» angelehnt wegen der «wärmenden» Eigenschaften des Wermuts.

Frühere Verwendung: Im Mittelalter begegnen wir dem Wermut im «Hortulus», dem Lehrgedicht des Abts Walafridus Strabus aus dem 9. Jahrhundert. Die wichtigsten Anwendungen waren jene als Wundmittel sowie gegen Cholera und Pest, Rheumatismus, Lähmungen, Gelbsucht, Wassersucht, Skorbut, Bleichsucht, Magenleiden, Frauenleiden und Epilepsie. Richter [7] schreibt über die Verwendung in künstlichen Wildbädern zur Provokation eines Badeausschlags. Auch Zieger [8] empfiehlt A. absinthium äusserlich zur Hautreizung, um die lokale Durchblutung zu verstärken. Nach Hahnemann [9] verstärkt das Kraut die Tätigkeit der Schlagadern, bringt zuweilen Schweiss hervor und heilt einige Hautausschläge durch äusseren Gebrauch wirksam. 
Abb. 3. Blätter von Salvia officinalis (Foto: Martina Föhn).

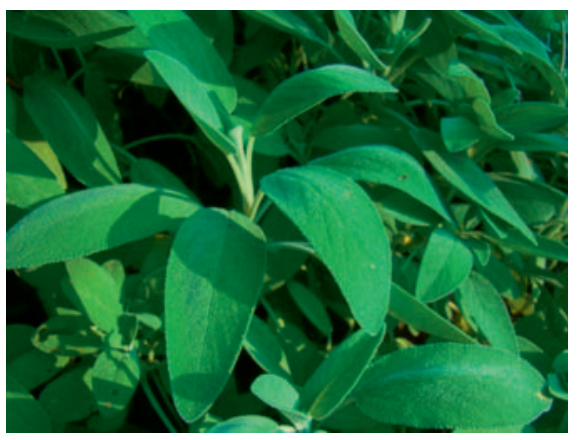

Heutige Verwendung: Wichtl [10] beschreibt Wermut heute zur Anregung des Appetits und als Digestivum, bei Menstruationsbeschwerden, bei Blutarmut und äusserlich bei schlecht heilenden Wunden und ekzemartigen Krankheiten. Wirkstoffe sind Bitterstoffe (Absinthin), Gerbstoffe und ätherisches Öl(Terpenewiez.B. $\beta$-Thujon). Herba Absinthii ist in der Schweiz heute offizinell.

\section{Salbine (Rezeptur 1 und 2) - Salvia officinalis L. -}

\section{Garten-Salbei}

Etymologie: Der Begriff «Salbine» wurde im 18. und 19. Jahrhundert in den Kantonen Luzern, Zürich, Zug, Glarus, Schaffhausen und Bern als volkstümlicher Name für S. officinalis L. (Abb. 3) benutzt [11].

Frühere Verwendung: In den Kräuterbüchern des 16. Jahrhunderts nimmt er als wundheilendes Mittel einen grossen Platz ein.

Heutige Verwendung: Nach Madaus [12] ist der Salbei gut gegen faule Geschwüre und wirkt blutreinigend, blutstillend und schweisshemmend. Salbei enthält ätherisches Öl mit Thujon, Cineol, Campher, Borneol und Bornylacetat, weiterhin Carnosolsäure, Rosmarinsäure, Flavonoide und Gerbstoffe. Salbei wirkt antimikrobiell, antibakteriell, fungizid, antiviral und adstringierend [13]. Äusserliche Anwendung findet Salbei heute bei Verletzungen, zur Reinigung und Wundheilung sowie zu Waschungen mit einem Wundwein [10]. Zieger [8] empfiehlt, Salbei aufgrund seiner belebenden Wirkung als Badezusatz am Morgen zu verwenden.

\section{Bethonie (Rezeptur 1) und Braune Bethonie (Rezeptur}

2) - Stachys officinalis L. - Gebräuchliche Betonie

Etymologie: Das Schweizerische Pflanzen-Idiotikon [11] verwendet die Glosse «Officinelle» oder «Braune Betonie « als schweizerdeutschen Mundart-Namen für Betonica officinalis L. oder S. officinalis (L.). Der Name «Betonie» stammt von alten Namen wie «Betonica» und «Vetonica» ab. «Betonica» soll von den spanischen Vettonis abgeleitet sein, die nach Plinius die Pflanze zum ersten Mal als Heilmittel benutzten.
Frühere Verwendung: Marzell [14] berichtet, dass die «Betonie» seit dem Beginn des 16. Jahrhunderts als «Braun Betonie» von der «Weiss Betonie» unterschieden wird. Bei der «Braunen Bethonie» handelt es sich um die rosa blühende Form von S. officinalis. Bis in das 19. Jahrhundert wurde sie sehr viel gegen Gicht, Migräne und Paralyse, zu verstärkter Expektoration und gegen Geschwüre eingesetzt. Nach Leonhart Fuchs [15] ist «Betonick seer gut zu den lamen glidern».

Heutige Verwendung: S. officinalis wird in der zeitgenössischen Heilkunde nur noch selten bei Paresen, Apoplexie, Taubwerden der Gliedmassen und schlechter Blutzirkulation [12] verordnet. Die Verwendung als Wundarznei kann heute mit dem hohen Gerbstoffgehalt (Betonicin, Stachydrin und Turicin) erklärt werden, durch den die Narbenbildung gefördert wird.

FischmÜntz (Rezeptur 1 und 2) und Rotte bachmüntz (Rezeptur 2) - Mentha aquatica L. - Wasserminze

Etymologie: Althochdeutsche Glossen sind «fiscminz», «mentastrum», «wismince», «vischminze» und «balsamita». Marzell [14] stellt «FischmÜntz» mit Mentha aquatica L., Mentha longifolia (L.) und Mentha x piperita L. gleich. In den meisten Arznei- und Botanikbüchern steht es für die einheimische M. aquatica («Wasserminze», auch «Bachminze» und «Rothe Münze»). Über die Identität der Minzen waren sich die «alten Wissenschaftler» nicht einig. Die Kräuterbücher des 16. Jahrhunderts nennen den Gebrauch von vier bis sechs verschiedenen «Müntzen». Brunfels [16] bemerkt, dass M. aquatica von etlichen Deutschen «Fischmüntz» und «Rotmüntz» genannt wird. Der Stängel ist rot gefärbt, und die Pflanze wächst gern an feuchten Orten, wo auch Fische leben. Bei der gleichzeitigen Nennung von «FischmÜntz» und «rotte BachmÜntz» in der Rezeptur 2 handelt es sich vermutlich um dieselbe Pflanze. Die doppelte Aufführung der Pflanze könnte als Fehler bei der Abschrift resultieren.

Frühere Verwendung: Hahnemann [9] gebraucht die Münzarten zu sogenannten Mutter- und Nervenbädern.

Heutige Verwendung: Nach Bock [4] werden alle Minzen zum Dämpfen, Erwärmen und Resoluieren (Resolution von Lateinisch «Lösung einer Krankheit, Zerteilung einer Entzündung, Lösung des Schleims bei Katarrhen») verwendet; sie sind geeignet für Schweissbäder, aber auch gegen Geschwulste und Geschwüre. M. aquatica enthält antiseptische und antifungale Monoterpen-Alkohole vom Linalool-Typ sowie expektorierende und antimikrobielle Monoterpen-Oxide (Menthofuran und 1,8-Cineol); in einigen Sippen können bis zu 46\% Sesquiterpene (z.B. Caryophyllen) vorkommen [17]. Die «Wasserminze» enthält kein Menthol. Sie dient heutzutage äusserlich zu Waschungen und zu Bädern [18]. 
Rott baadkrautt (Rezeptur 1) und Wohlgemut

(Rezeptur 1) und Rotte constantz (Rezeptur 2) -

Origanum vulgare L. - Dost

Etymologie: Andere Namen für den einheimischen, recht häufigen Origanum vulgare sind Badkraut, Brauner Dost, Dosten, Echter Dost, Oregano, Schusterkraut, Spanischer Hopfen, Wilder Majoran, Wohlgemut und Hopfenkraut. Das «Rott Baadkrautt» hat seinen Namen der Verwendung zu Bädern zu verdanken.

O. vulgare wird auch «Wolgemut» genannt, vielleicht wegen seines angenehmen Geruchs und gewürzhaften Geschmacks. In Bern und im Zürcher Oberland bezeichnete man es als «Wolgemuet».

«Costentz», «Kostenz», «Grosser Chostez» und «Rotten Kosten» sind Glossen, die vermutlich aus dem Mittellateinischen "costus» entstanden sind. Sie wurden nach Marzell [14] unter anderem in den Kantonen Aargau, Bern, Vier Orte und Zürich verwendet. Im Gegensatz zum Namen «Kleiner ChosteZ» (Thymus serpyllum) wurde der Name «Grosser Chostez» für O. vulgare verwendet. Bei «Rotten Kosten» oder «Rote Koste» ist das Wort identisch mit «Koste» und bedeutet «Quaste, Büschel», was dann auch der Grundbedeutung von «Dost» entsprechen würde.

Mit der Nennung von «Rott Baadkrautt» und «Wohlgemut» in der Transkription der Rezeptur 1 ist offenbar beide Male O. vulgare gemeint, was nicht sinnvoll erscheint. Interessanterweise ist die Glosse «Rott Baadkrautt» in der Abschrift in Hallwyl nicht mehr aufgeführt. Möglicherweise hat der Verfasser dieser Abschrift bemerkt, dass die Pflanze zweifach genannt wird und hat einen Namen daher weggelassen. In der Rezeptur 2 wird weder die Glosse «Rott Baadkrautt» noch «Wohlgemut» verwendet. Hier wird «Rotte constantz» genannt. Diese Glosse wird als Synonym für O. vulgare verwendet und ist eventuell auf sprachliche Anpassungen zurückzuführen.

Frühere Verwendung: Als nervenstärkend bereits im Arzneischatz des Hippokrates und Paracelsus erwähnt, wird der «Dost» bei Madaus [19] als entzündungswidriges Mittel und gegen Asthma angewendet. Er empfiehlt ihn äusserlich gegen roten Aussatz. Ausserdem schreibt er der Pflanze eine spasmenlösende Wirkung zu. Nach Hahnemann [9] ist das Kraut grösstenteils bloss als ein äusserliches, zerteilendes, nervenreizendes Mittel angewendet worden.

Heutige Verwendung: O. vulgare enthält ätherisches Öl mit den Bestandteilen Carvacrol und Thymol [10] sowie Gerbstoffe und wird heutzutage äusserlich in Gurgelwässern und für Bäder eingesetzt. Von Bruchhausen et al. [13] nennen die Verwendung bei Rheuma und Skrofulose und beschreiben ihn als schweisstreibend, beruhigend, krampflösend und antimikrobiell.

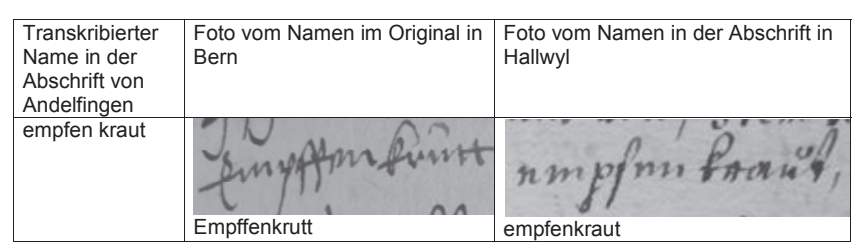

Abb. 4. Vergleich der verwendeten Glossen «Empfen kraut» in Rezeptur 1.

\section{Rott bugcken (Rezeptur 1) und Rott buggelen}

(Rezeptur 2) - Artemisia vulgaris L. - Gemeiner Beifuss Etymologie: Von den Glossen «Rott bugcken» und «Rott buggelen» lassen sich mehrere Kräuter ableiten. Da aber in einer weiteren Rezeptur Burkhards «Ein anders bewÖrtes stuck» «rott Buggelen» mit «Byfuss» gleichgesetzt wird, lässt sich die Identität auf Wermut zurückführen. Das Wort «buc» bzw. «bucckes» wurde in der mittelhochdeutschen Sprache für Schlag, Stoss, Sturz und Beifuss verwendet. Im Altfranzösischen heisst Beifuss auch «bocle», auf Lateinisch «buccula» [6]. Der deutsche Name Beifuss (althochdeutsch «pīpō3», mittelhochdeutsch «bibuo3») wird von dem althochdeutschen Verb «bōzen», d.h. «stoßen, schlagen», abgeleitet. Von «bugila» und «buggela» wurde im 10. Jahrhundert gesprochen, während «bucca», «bugga», "puggo» und «bugge» aus dem 11.-14. Jahrhundert stammen, und «bukel» wurde im 14. Jahrhundert verwendet [14]. In Stoffler [20] zitiert Brunfels: «Wir Teutschen nennen es Beyfuss, Buck.» Das Schweizerische Pflanzen-Idiotikon datiert die Benennung von A. vulgaris mit «Rot Buckele» auf das 16./17. Jahrhundert.

Frühere Verwendung: Bock [4] verwendet den Beifuss oder «Bucken» als nützliches Frauenkraut. Beifussblumen setzt er bei erkalteten, lahmen Adern ein.

Heutige Verwendung: Das Kraut enthält ätherisches Öl mit Monoterpenen. Daneben kommen Cumarine und Sesquiterpen-Bitterstoffe vor. Die Erfahrungen der praktisch tätigen Wundärzte hat sich durch die heute wissenschaftlich bewiesene antibakterielle und antimikrobielle Wirkung bestätigt [7]. Madaus [21] hält ihn für ein besonders gutes Wund- und Mutterkraut.

\section{Empfen kraut (Rezeptur 1) und Špfen kraut}

(Rezeptur 2) - Apium graveolens L. - Sellerie oder

Hedera helix - Efeu?

Für die Glosse «Empfen kraut» wurden in der Literatur keine treffenden Begriffe gefunden. Daher ist die Identifizierung nicht eindeutig. Möglicherweise wurden Buchstaben abgeändert (ampfer - empfen) oder hinzugefügt (epfen - empfen) (Abb. 4).

Für die Glosse «Špfen kraut» werden in dem Originalarzneibuch, den Abschriften und der Transkription verschiedene Wörter verwendet (Abb. 5). 


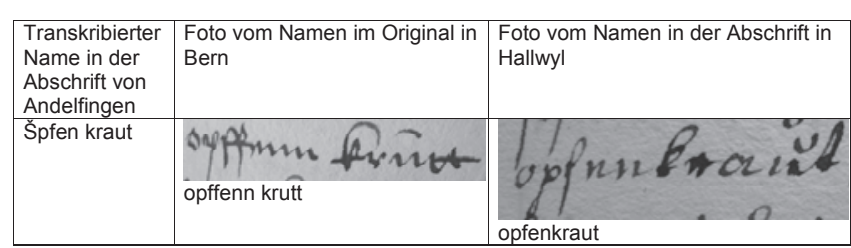

Abb. 5. Vergleich der verwendeten Glossen von «Špfen kraut» in Rezeptur 2.

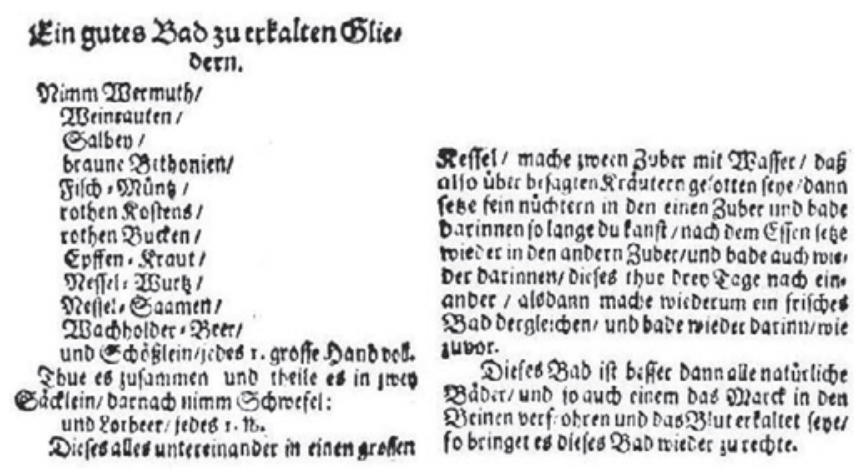

Abb. 6. Rezeptur aus dem Arzneibuch eines Haus- und Landarztes, das 1727 in Nürnberg geschrieben worden ist.

In Burkhards Arzneibuch wird das «š» für «ö» verwendet, z.B. in «cšrper», oder für «ä», wie z.B. in «bewšrt». Die Glosse «Špfen kraut» in der Transkription in Andelfingen heisst dann wohl «Öpfen kraut» oder «Äpfen kraut». Zu «Špfen kraut» und «Opfenkraut» wurden bei der Literaturrecherche keine entsprechenden Namen gefunden. Die Weglassung eines «h» am Wortbeginn könnte auf den Ursprung Hopfenkraut (siehe auch «rott Baadkraut» - O. vulgare) zurückführen. Es könnte auch eine Umformung von «e» $\mathrm{zu}$ «o» stattgefunden haben. Dann würde die Glosse auf «Epfen kraut» führen, das wiederum ein Synonym zu «Empfen kraut» sein könnte. Das Arzneibuch eines Haus- und Landarztes von 1727 in Nürnberg führt unter demselben Titel eine Rezeptur auf, die auch inhaltlich der Rezeptur 2 sehr ähnlich ist (Abb. 6). Hierin wird die Glosse «Epffen kraut» genannt. Dies lässt darauf schliessen, dass mit «Empfen kraut» in Rezeptur 1 und «Špfen kraut» in Rezeptur 2 ein und dieselbe Pflanze gemeint sein könnte.

Eine grosse Affinität lässt sich bei den Glossen «Empfen kraut» und «Špfen kraut» zu den Pflanzen Apium graveolens und Hedera helix feststellen. Die Glossen können jedoch nicht eindeutig identifiziert werden.

\section{Apium graveolens L. - Sellerie}

Etymologie: «Epf» heisst nach Grimm und Grimm [22] «Eppich», althochdeutsch «wilder Eppi». Ähnliche Glossen sind «Ephi», «Effi», «Effe», «Epfe», «Ephkrut», «Epff», «Eppe», «Hepfen» und «Hepffen».
Frühere Verwendung: Madaus [21] schreibt, dass der «Epffich»-Samen Wunden säubere, und zählt ihn zu den erwärmenden Samen. A. graveolens, auf Deutsch Sellerie, wird bei Hautkrankheiten wie Urtikaria, Flechten und Perniones sowie bei Gicht und Rheuma (hier auch äusserlich als Bad) empfohlen. Tabernaemontanus [23] unterscheidet den "gemeinen Eppich», welches der «rechte» ist, vom «wahren Garteneppich», der Petersilie. Er ist der Meinung, dass die Heilkundigen A. graveolens fälschlicherweise für das "wahre Apium» verwendet haben. Wundärzte setzten ihn bei allerlei Krankheiten ein: zur Reinigung von Wunden, bei Geschwüren, alten Schäden, Krebs, Nerven- und Sennadern, Entzündung und hitziger Geschwulst der Brust sowie gegen den «Kaltseych» oder die «Harnwindt».

Heutige Verwendung: A. graveolens enthält ätherisches Öl mit Cymol, Santalol, Limonen und Pinen. Die heutige Verwendung beschränkt sich nach Wagner [24] auf den Einsatz als Stomachikum, Karminativum, Diuretikum und Emmenagogum.

Hedera helix L. - Efeu

Etymologie: Gebräuchliche Glossen sind «Epfa», «Epphae» usw.

Frühere Verwendung: Efeu ist ein gutes Wundmittel. Äusserlich wurde er in der Volksmedizin gegen Geschwüre, Entzündungen und Brandwunden angewendet. Kölbl [25] empfiehlt Efeublätter in Form von Bädern bei Flechten, Krätze und Frostbeulen. Nach Madaus [19] verursacht Hedera einen stark juckenden urtikariellen Ausschlag. Bei Brandwunden und eiternden Wunden ist die äusserliche Anwendung des Efeus von Nutzen. Hahnemann [9] setzt die Blätter gegen Gichtschmerzen ein. Auch das Epheuharz wurde äusserlich als wundheilendes Mittel verwendet.

Heutige Verwendung: H. helix beinhaltet ein Saponingemisch mit Hederacosid. Dieses wirkt expektorierend und spasmolytisch. Die Efeu-Saponine wirken auch antimykotisch und antibakteriell. Wagner [24] verwendet es heute gegen Bronchialkatarrh, Keuchhusten und Asthma. Frische Efeublätter und der Blattsaft können wegen des Hautallergens Falcarinol allergische Kontaktdermatitiden verursachen.

\section{LYbstickel (Rezeptur 1 und 2) - Levisticum officinale} Koch - Liebstöckel

Etymologie: Levisticum officinale leitet sich aus dem Mittellateinischen «Libisticum» bzw. «Lubisticum»ab.

Frühere Verwendung: Äusserlich wurde Liebstöckel als Bäderzusatz bei schlecht heilenden Wunden und Eiterungen und bei Erbgrind sowie weiteren Gebresten verwendet [19]. Bock [4] bezeichnet Liebstöckel als ein «wohlrie- 
chendes Badkraut» und schreibt: «Dempff und wasser bäder von beiden Liebstöckel gewachsen zubereit fordern und treiben den Harn, den Stein, der Frawen Krankheit und erwörmen alle Innerliche glider.»

Heutige Verwendung: Liebstöckel enthält Cumarin, Umbelliferon, Bergapten und Psoralen [24]. Die Furanocumarine entfalten stark photosensibilisierende Eigenschaften und verursachen nach Sonneneinstrahlung eine Bläschenbildung auf der Haut [24]. Nach Richter [7] beruht der Einsatz in der Wundbehandlung wohl auf dem Gehalt an ätherischem Öl in den Blüten. Es wird heute als Diuretikum bei entzündlichen Erkrankungen der ableitenden Harnwege, zur Vorbeugung bei Nierengriess, bei ödematösen Schwellungen in den Füssen, als Stomachikum, Karminativum, Emmenagogum und schleimlösendes Mittel bei Katarrhen der Luftwege sowie bei Verdauungsbeschwerden, Völlegefühl und Sodbrennen angewandt [10].

\section{Neslen wurtz und nesslen sammen (Rezeptur 1)} sowie Neßlen wurtz und sammen (Rezeptur 2) Urtica dioica L. - Grosse Brennnessel

Etymologie: Nessel stammt vom Mittelhochdeutschen «Nezzel». Erbar und Zimmermann [26] erläutern, dass «Urtica maior» oder «Grosenezzil» für Urtica dioica $\mathrm{L}$. und «Eyterneszel» für Urtica urens L. stehen.

Frühere Verwendung: Nach Madaus [12] ist das Schlagen rheumatischer oder gelähmter Glieder mit Brennnesseln besonders beliebt, wobei es sich aber lediglich um eine Hautreizung durch das Gift der Nesselhaare zur Provokation eines Badeausschlags handelt. Zieger [8] erklärt, dass die behandelten Körperzonen dabei kräftig durchblutet werden; gleichzeitig nimmt die Haut entgiftende Wirkstoffe auf, die von innen her antirheumatisch wirken. Die Nessel wirkt gegen krebsartige Geschwüre, brandige Wunden, Furunkel, Geschwülste, Lähmungen, Drüsenanschwellungen und Verrenkungen.

Heutige Verwendung: U. dioica enthält antiphlogistisch wirkendes Agglutinin [10]. Ferner wirkt es diuretisch, immunologisch und fungistatisch. Nach Richter [7] enthalten Samen fettes Öl, weswegen sie «einen unterstützenden Effekt auf Wundheilung sowie bei Hautleiden haben».

\section{Reckholterbere (Rezeptur 1) und Reckholter bere}

(Rezeptur 2) - Juniperus communis L. s. l. - Wacholder Etymologie: Marzell [14] führt die Entstehung des Begriffs «Reckholder» auf «Weckholder» und «Quekholder» zurück. Der erste Teil bezieht sich auf «recken», indem der Wacholder seine Zweige überall hin ausbreitet. Im Schweizerdeutschen ist der erste Bestandteil nicht selten auf «rauken», «räuken», «räuchern» und «rauchen» umgedeutet, da Zweige und Beeren des Wacholders zum Räuchern verwendet wurden.

Frühere Verwendung: Bei Madaus [19] waren die Beeren schon im Altertum als Antiseptikum bekannt. Die Beeren finden Verwendung als Wundmittel und gegen Lähmungen, Gicht, Rheumatismus, Nervenschwäche, chronische Hautausschläge, Skabies und Herpes.

Heutige Verwendung: Wacholder enthält ätherisches Öl (Pinen, Sabinen, Myrcen, Limonen, Terpinenol). Nach Von Bruchhausen et al. [13] wirkt er diuretisch, choleretisch und antimikrobiell. Bei lokaler Anwendung besitzen Wacholderbeeren aufgrund ihres Gehalts an $\alpha$ - und $\beta$-Pinen eine hautreizende und durchblutungssteigernde Wirkung, weswegen sie bei Gicht und Rheuma zum Baden eingesetzt werden.

\section{Schwebel (Rezeptur 1 und 2) - Schwefel}

Etymologie: Beim «Schwebel» handelt es sich eindeutig um den Schwefel.

Frühere Verwendung: Die Schwefelbalsame sind nach Hahnemann «äusserst erhitzende Substanzen, die die Alten bei innern und äussern Geschwüren mit schlaffer, kalter Körperbeschaffenheit» verabreichten [9].

Heutige Verwendung: Zur Bereitung eines Schwefelbades wird kolloidaler Schwefel verwendet [5]. Schwefelbäder sind zur Verbesserung der Hautdurchblutung, bei entzündlich-rheumatischen Erkrankungen, degenerativen Gelenkerkrankungen, Muskelrheumatismus sowie juckenden, schuppenden und entzündlichen Erkrankungen der Haut (Ekzeme, Schuppenflechte usw.) angezeigt.

\section{Lorberi (Rezeptur 1) und Lorbonen (Rezeptur 2) -}

Laurus nobilis L. - Lorbeer

Etymologie: Das Deutsche entlehnt «Laurus» dem Althochdeutschen «Lōr-boum» und «Lōr-beri». Dabei bezeichnet «Lōrberi» zunächst die Frucht (Beere) des Baums.

Frühere Verwendung: Nach Hahnemann [9] regen die Blätter den Blutkreislauf an. Das Öl ist äusserlich aufgebracht ein gutes Nerven ermunterndes Mittel bei Schwäche der Gelenke, Lähmung und Taubheit. Als Einsatzgebiete für Lorbeeröl erwähnt Richter [7] erlahmte Adern und Glieder wie auch das «Schwinden» (Atrophie). Bock [4] setzt Lorbeeren gerne bei kältebedingten Leiden wie dem «Schwinden» oder bei Verrenkungen und Zerrungen des Bewegungsapparats ein.

Heutige Verwendung: Das Öl der Früchte ist ein Gemisch von ätherischem Öl (Cineol, Terpineol, $\alpha$ - und $\beta$-Pinen und Citral, aber auch Zimtsäure und ihre Methylester) sowie fettem Öl. Das Öl enthält auch einige Sesquiterpenlactone, die für die allergieauslösenden Eigenschaften von Lorbeer verantwortlich gemacht werden. 
Wegen der antiseptischen und hautreizenden Wirkung der ätherischen Öle findet es Anwendung in der Traumatologie, Dermatologie und Ophthalmologie.

\section{Weißer augstein (Rezeptur 1) - Bernstein}

Etymologie: «Agstein», «Agtstein» und «Augstein» ist nach Paracelsus Bernstein [27]. «Agstein» ist die frühneuhochdeutsche Bezeichnung für das althochdeutsche Wort «Bernstein».

Frühere Verwendung: Bernstein, das fossile Harz von ca. 55 Millionen Jahre alten Nadel- und Laubbäumen, kommt in verschiedenen Farben vor. Der seltene weisse Bernstein erhält seine Farbe durch die vielen Lufteinschlüsse im versteinerten Harz. Lonitzer (1528-1586) empfiehlt in seinem Werk «Naturalis historiae opus novum» (1551-1555) die weisse Form «wegen des guten Geruchs», den der Bernstein beim Räuchern produziert. Ab dem 16. Jahrhundert wurde Bernstein in den Apotheken verkauft. Im 17. Jahrhundert wurden auch verschiedene neue Zubereitungen beschrieben, z.B. Succinum raspatum (geraspelter Agtstein) sowie Succinum praeparatum album als fein zerriebener, weisser Bernstein. Ersch und Gruber [28] beschrieben verschiedene Zubereitungen wie Wundbalsam sowie Dampfbäder gegen hysterische Beschwerden, Krämpfe, Hüft- und Wadenschmerzen, rheumatische und andere Schmerzen, Schwindsucht, Lungenleiden und viele andere Gebrechen.

Heutige Verwendung: Bernsteinsäure sowie Bernsteinöl wirken auf der Haut hyperämisierend. Bernstein wird heute in homöopathischer Verschüttung gegen rheumatische Schmerzen und Nervenschmerzen bei Degenerationsschäden der Gelenke beschrieben. Ansonsten ist Bernstein aber aus den Pharmakopöen des 20. Jahrhunderts verschwunden [29].

Da Bernstein in heissem Wasser unlöslich ist, müsste er in der Rezeptur vorher aufgelöst worden sein, möglicherweise in Honig oder Lavendelöl. Da zu dieser Zeit Destillationen bereits bekannt waren, wäre die Verwendung der dabei entstehenden wasserlöslichen Bernsteinsäure möglich. Nicht auszuschliessen ist auch die Verwendung von Ersatzprodukten für den seltenen weissen Bernstein. Infrage kommen z.B. Weihrauchkörner, die von Fichten und Tannen gesammelt wurden.

\section{Weißer wierauch (Rezeptur 1) - Harz von Boswellia sp. - Weihrauch}

Etymologie: Richter [7] zeigt verschiedene Synonyme für Weihrauch auf, z.B. «Weiroch», «Weirach», "Weirauch» und «Wiroch». Nach Von Bruchhausen et al. [13] stammt Weihrauch (Olibanum) aus Somalia, Südarabien und Äthiopien und ist das aus Einschnitten in die Bäume von Boswellia carteri BIRDW. und Boswellia frereana
Abb. 7. Ruta graveolens (Foto: Martina Föhn).

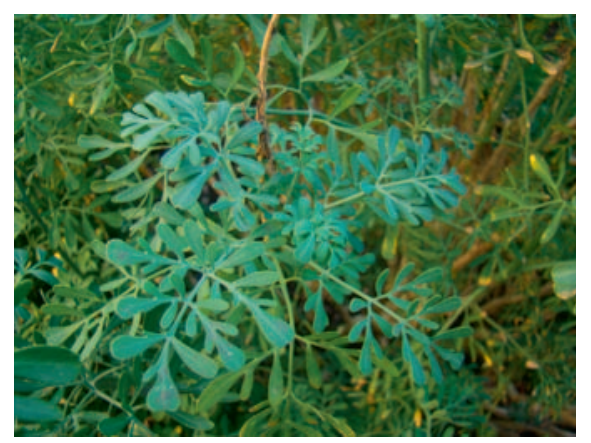

BIRDW. ausgetretene erstarrte Gummiharz. Weihrauch bildet unregelmässige Körner, die gelblich, gelbrötlich oder bräunlich, aussen weiss bestäubt sind.

Frühere Verwendung: Hahnemann [9] verwendete den Weihrauch als äusseres Wundheilmittel und zum Räuchern.

Heutige Verwendung: Bereits Madaus [12] kennt Weihrauch als adstringierendes, erwärmendes und zum Heilen von Wunden benutztes Mittel. Vollmuth [30] verwendet Weihrauch in vielen spätmittelalterlich-frühneuzeitlichen Rezepten der Traumatologie. Den Inhaltsstoffen (ätherisches Öl und Harzsäuren) wurden sowohl analgetische und antiinflammatorische als auch immunsuppressive und hepatoprotektive Eigenschaften nachgewiesen. In der klassischen europäischen Naturheilkunde wird der Weihrauch hauptsächlich zur Linderung von rheumatischen Erkrankungen eingesetzt. Bereits seit Jahrhunderten wird er in der traditionellen ayurvedischen Medizin Indiens bei Entzündungen, Tumoren und Rheumatismus verwendet.

\section{Rautte (Rezeptur 2) - Ruta graveolens L. - Raute}

Etymologie: Der deutsche Name «Raute» ist nach Marzell [14] aus dem lateinischen «Ruta entstanden.

Frühere Verwendung: Die Raute (Abb. 7) war bereits im «Capitulare de villis» des Kaisers Karl des Grossen (747-814), im Herbularius des St. Galler Klosterplans (820) und im Garten des Walahfrid Strabo (808-849) als Heilkraut gepflanzt [20]. Marzell [14] verwendet sie äusserlich gegen Gelenkschmerzen und Ausschlag. Weiterhin setzt er sie in Form von Dämpfen, Umschlägen und Bädern bei kalten Geschwülsten, Lähmungen und Augenschwäche ein. Diese Wirkung beschreibt bereits Bock [4] mit der Entstehung roter Blasen bei äusserlicher Anwendung der Raute.

Heutige Verwendung: Neben dem ätherischen Öl enthält das Kraut Flavonoide (Rutin), Alkaloide und Cumarine. Die Furocumarine sind für die photoallergischen Reaktionen verantwortlich. Anfang des 20. Jahrhunderts wurde sie als ausgezeichnetes Muskelmittel bei rheumatischen Gliederschmerzen und Gelenkrheumatismus sowie als anregend auf die Tätigkeit des Uterus beschrieben. 


\section{Bestimmung der Masse und Gewichte}

Ein Lot entspricht ungefähr $15 \mathrm{~g}$, ein Vierling in etwa $125 \mathrm{~g}$ [31]. Die Volumenangabe «Handvoll» ist nach Seraphim [32] ein grobes Apothekermass bei Kräutern und entspricht einem Lot. Der Haller oder Heller war ursprünglich ein Pfennig aus der Stadt Schwäbisch Hall und entspricht nach Ritter [33] einem Hellergewicht von ca. 3,75 g.

\section{Interpretation der Rezepturen}

$\mathrm{Zu}$ den verwendeten Pflanzenteilen werden in den beiden Rezepturen keine detaillierten Angaben gemacht mit Ausnahme der Nesselsamen und -wurzeln und der Lorbeeren. Auch zur Sammelzeit der Kräuter werden keine Aussagen getroffen. Die meisten der identifizierten Pflanzen werden nach heutigen Literaturangaben im Juli und August gesammelt. Unklar ist, ob die Pflanzen in frischem oder getrocknetem Zustand verwendet werden sollen. Die Mengenangaben erlauben eine relativ präzise Dosierung. Unklar ist auch, ob die Kräuter im Badewasser verbleiben oder herausgenommen werden. Bei den Bädern handelt es sich vermutlich um absteigende Bäder, bei denen die Temperatur durch Erkalten kontinuierlich abnimmt. Das Wasser dient zur Reinigung von Schmutz und auch der Abfuhr von schädlichen Säften. Weiterhin können im Badewasser gelöste Inhaltsstoffe durch die in der Wärme geöffneten Hautporen resorbiert werden [5]. Die verwendeten Pflanzen werden als warme, trockene Pflanzen bezeichnet, die in der Lage sind, zu «erwärmen». Die Wärmeeinwirkung und Hautreizung mit hyperämisierenden Stoffen führen zu einer Erhöhung der Abwehrzellen und Immunstoffe. Einige Bestandteile in Burkhards Rezepturen bewirken einen Hautausschlag, z.B. «reckholter». Der Badeausschlag könnte eine für die Heilung erwünschte Reaktion sein [34]. Die Patienten sassen früher oft täglich etwa 10-12 Stunden im Bad, bis die Haut wund war und zu eitern begann. Diese Wirkung war durchaus erwünscht, denn man glaubte, dass das heilende Wasser nur durch die offene Haut richtig in den Körper eindringen könne [35]. Am ehesten handelt es sich in diesen Rezepturen um ein Dreiviertel- oder Vollbad. Beim warmen Dreiviertel-Bad mit einer Temperatur von $36-38^{\circ} \mathrm{C}$ werden auch Krankheiten wie Katarrhe der oberen Luftwege, Bronchitis und Knocheneiterungen behandelt. Zu Zeiten Burkhards wurde oft ein geflochtener Korb oder ein Baldachin aus Stoff über dem Bad installiert, um die Wärme und die Dämpfe länger zu konservieren [2].

\section{Interpretation der Rezeptur 1}

Es wird nicht erwähnt, ob aus den Rezepturbestandteilen ein Dekokt oder ein Infus hergestellt wird. Als Indi- kation dieser Rezeptur werden im Titel «allerlei Gebrechen» angegeben. Von zeitgenössischen Wissenschaftlern Burkhards, wie Otto Brunfels, Hieronymus Bock, Leonhart Fuchs, Tabernaemontanus und Adamus Lonicerus, aufgeführte Indikationen für die in Rezeptur 1 verwendeten Pflanzen sind häufig rheumatische Erkrankungen, Hauterkrankungen, Wunden, Schmerzen, Steifheit, aber auch Lähmungen, Gliederschwund, Frauenkrankheiten und Schlag. Aus dem Schwefel erfolgt im Wasser oder durch organische Substanzen der Haut die Freisetzung von Schwefelwasserstoff, der relativ leicht durch die Haut aufgenommen wird [5]. An die Haut lagert sich auch Sulfid an, das noch über 24 Stunden lang eine gewisse Nachresorption ermöglicht. Deshalb soll nach Burkhard kein Wasser zum Abwaschen verwendet werden. Nüchtern soll jeweils gebadet werden, damit die Körperstoffe ausgeschwitzt werden können. Weisser Augstein und weisser Weihrauch werden als Desinfektionsmittel zum Badewasser gegeben. Beide haben zusätzlich eine arzneiliche Wirkung bei rheumatischen Erkrankungen und Polyarthritis sowie als Wundheilmittel.

\section{Interpretation der Rezeptur 2}

Auch dieses Bad wird wie Rezeptur 1 für vielerlei Gebrechen empfohlen, besonders jedoch für erkaltete Glieder, also «wenn das Mark in den Beinen erfroren oder das Blut erkaltet ist». So wie Ryff [36] mit dem Begriff «Glyder» nicht nur Arme und Beine, sondern äussere und innere Organe meint, behandelt Burkhard mit dieser Rezeptur vermutlich ebenso den gesamten Körper. Die Bemerkung «nach dem Essen» deutet darauf hin, dass das erste Bad nüchtern, das zweite Bad nach dem Essen erfolgt. "So lange man mag» bedeutet vermutlich, bis das Badewasser unangenehm kühl wird. Diese Badekur wird 3 Tage mit Kräuterzusatz als Dekokt durchgeführt; anschliessend badet man ohne Kräuterzusatz. Die Indikationen beziehen sich vor allem auf die Blutgefässe und Nerven in den Gliedmassen und im Körper. Dies könnten Krankheiten wie Gicht, Rheuma und Lähmung, z.B. nach Schlaganfall, sein. Auch Durchblutungsstörungen, wie sie im Mittelalter beim Antoniusfeuer infolge einer Vergiftung von mit Mutterkorn infiziertem Getreide auftraten, könnten hiermit behandelt worden sein.

\section{Fazit}

Die Rezepturen 1 und 2 ähneln sich sehr stark. Viele Pflanzen von Rezeptur 1 werden auch in der Rezeptur 2 verwendet. Auch die beschriebenen Anwendungen sind sehr ähnlich. Dies könnte auf die Abwandlung einer Rezeptur hindeuten, z.B. durch Hinzufügen oder Weglassen von einigen Materialien. Die Rezepturen könnten aus un- 
terschiedlichen Sprachregionen stammen. Möglicherweise wurden sie auch zu verschiedenen Zeiten niedergeschrieben. Beispielsweise stammt die Glosse «Lorberi» aus dem Althochdeutschen, während «Lorbonen» seinen Ursprung im 16. Jahrhundert hat. Eventuell wurden die Rezepturen durch das mehrmalige Abschreiben über einen längeren Zeitraum unter Weiterentwicklung der Sprache und Schrift verändert. Manche Glossen könnten beim Abschreiben falsch interpretiert worden sein. Eine eindeutige Identifikation der verwendeten Pflanzen und Stoffe ist nicht in allen Fällen möglich. Zum Teil herrschte unter den Wissenschaftlern zur Zeit Burkhards Uneinigkeit über die Identität einer Pflanze. Botanische Beschreibungen und Zeichnungen waren ungenügend oder nicht eindeutig. Für eine Pflanze gab es in den verschiedenen Sprachregionen oft Synonyme. Da Rezepte auch aus anderen Regionen oder Ländern stammen können, sind die archäobotanische Analyse zum Vorkommen der Pflanzen in der Umgebung des Schlosses Hallwyl sowie vegetationskundliche Studien nicht aussagekräftig genug. Nachforschungen über den Briefwechsel von Burkhard III. könnten wichtige Indizien für die nähere Bestimmung der Pflanzen und Stoffe enthalten: z.B., woher die Rezepturen stammen (Region, Sprachraum), in welchem Jahr sie zum ersten Mal niedergeschrieben worden sind und aus welcher Rezeptsammlung sie stammen. Linguistische Studi- en könnten die Herkunftsbestimmung der Rezepturen erleichtern und nähere Aufschlüsse über die Identität der verwendeten Kräuter geben, z.B. bei der Identität von «Špfen kraut». Die interdisziplinäre Zusammenarbeit von Botanikern, Pharmazeuten und Sprachwissenschaftlern könnte durch die Beantwortung noch offener Fragen zu einer Vervollständigung der Analyse führen. Diese Arbeit leistet einen Beitrag zur Analytik des Arzneibuchs von Burkhard III. von Hallwyl.

\section{Dank}

Mein besonderer Dank gilt Barbara Frei Haller, die mir diese Arbeit überhaupt ermöglichte. Thomas Frei und Jeannette Strebel möchte ich für die Erlaubnis zum Fotografieren im Schloss Hallwyl danken. Dank geht auch an Maja Dal Cero für die Durchsicht des Manuskripts.

\section{Disclosure Statement}

Martina Föhn, pharmazeutisch-technische Assistentin und Dipl. Hortikulturingenieurin FH, ist wissenschaftliche Mitarbeiterin der Zürcher Hochschule für Angewandte Wissenschaften (ZHAW) in Wädenswil und Projektleiterin Zierpflanzen bei ProSpecieRara. Die vorliegende Arbeit ist die überarbeitete Version einer Abschlussarbeit des Zertifikatstudiengangs «Ethnobotanik und Ethnomedizin» der Universität Zürich.

Für die Autorin bestehen keinerlei Interessenkonflikte.

\section{Literatur}

1 Kiby U: Die Badekultur, ein Vergnügen der Menschheit seit der Antike; in Grohe $\mathrm{H}$ : Badewonnen. Köln, DuMont, 1993, pp 6-90.

2 Ausstellung «Forschen und Heilen», Schloss Hallwyl (besucht am 10.7.2009).

3 Frei Haller B: Das recht Hallwylisch wundtrannck; in 900 Jahre Leben auf Schloss Hallwyl, Band 2. Bern, Stämpfli, 2005, pp 69-90.

4 Bock H: Kreutterbuch, Reprint vom Original 1577. Grünwald, Konrad Kölbl, 1964.

5 Gillert O, Rulffs W: Hydrotherapie und Balneotherapie, ed 11. München, Pflaum, 1990.

6 Lexer M: Mittelhochdeutsches Taschenwörterbuch, ed 38. Stuttgart, S. Hirzel, 1992.

7 Richter C: Phytopharmaka und Pharmazeutika in Heinrich von Pfalzpaints «Wündärznei» (1460), Dissertation. Würzburg, Bayerische Julius-Maximilians-Universität, 2003, pp 172-173.

8 Zieger R: Das grosse Heilkräuter-Lexikon. Zürich, Buch-Vertriebs-Gesellschaft, 1978.

9 www.heilpflanzen-welt.de/buecher/HahnemannApothekerlexikon/ (zugegriffen am 01.09.2009).

10 Wichtl M: Teedrogen und Phytopharmaka, ed 5. Stuttgart, WVG, 2009.

11 Durheim CJ: Schweizerisches Pflanzen-Idiotikon, unveränderter Neudruck von 1856. Bern, Huber \& Comp. (Körber), 1983.

12 Madaus G: Lehrbuch der biologischen Heilmittel, Band III, Nachdruck von 1938. Leipzig, Thieme, 1979.

13 Von Bruchhausen F, Hager H, Dannhardt G, Ebel S: Hagers Handbuch der Pharmazeutischen Praxis, ed 5, Band 1-9. Berlin, Springer, 1995.
14 Marzell H: Wörterbuch der deutschen Pflanzennamen, Band 1-5, fotomechanischer Nachdruck der Erstausgabe 1958. Köln, Parkland, 2000.

15 www.waimann.de/capitel/projekt.html (zugegriffen am 18.10.2009)

16 Brunfels O: Kreuterbuch contrafayt. Strassburg, Hans Schotten, 1534

17 Bolli R: Pfefferminze und Pfefferminzöl. Phytotherapie 2003;5:14-18.

18 www.natwiss.ph-karlsruhe.de/GARTEN/ material/steckbrief/heilpflanze/wasser-minze_ ph-ka.pdf (zugegriffen am 07.02.2010).

19 Madaus G: Lehrbuch der biologischen Heilmittel, Band II, Nachdruck von 1938. Leipzig, Thieme, 1976.

20 Stoffler H-D: Der Hortulus des Walahfrid Strabo, ed 6. Stuttgart, Jan Thorbecke, 2000, pp 68-69.

21 Madaus G: Lehrbuch der biologischen Heilmittel, Band I, Nachdruck von 1938. Leipzig, Thieme, 1976.

22 Grimm J, Grimm W: Deutsches Wörterbuch von Jacob Grimm und Wilhelm Grimm. Leipzig, S. Hirzel, 1971.

23 www.kraeuter.ch (zugegriffen am 20.10.2009)

24 Wagner H: Pharmazeutische Biologie, ed 2, Band 2: Drogen und ihre Inhaltsstoffe. Stuttgart, Gustav Fischer, 1982.

25 Kölbl K: Kölbl’s Kräuterfibel, ed 8. München, Reprint-Verlag Konrad Kölbl, 1972.
26 Erbar C, Zimmermann K: Der Codex Palatinus germanicus 539 - eine Pflanzenliste aus dem 15. Jahrhundert. Heidelberg, Universität Heidelberg, 2009.

27 www.paracelsus.uzh.ch (zugegriffen am 24.10.2009).

28 Ersch JS, Gruber JG: Allgemeine Encyclopädie der Wissenschaften und Künste. Leipzig, Johann Friedrich Gleditsch, 1822.

29 www.gemline.ch/gemmline\%20untermenu/ Bernstein/bernstein $\% 20 P D F /$ Bernstein $\% 20$ Geschichte.pdf (zugegriffen am 19.10.2009).

30 Vollmuth R: Traumatologie und Feldchirurgie an der Wende vom Mittelalter zur Neuzeit. Stuttgart, Franz Steiner, 2001.

31 www.ahne1.de/altemasse.htm (zugegriffen am 22.02.2010).

32 Seraphim A: Altpreussische Monatsschrift, Band 43. Königsberg, Thomas \& Oppermann, 1906, p 504.

33 Ritter G: Transkription von mittelalterlichen Quellentexten computergestützt üben. Zürich, Universität Zürich, 1992.

34 Obes DJ: Philipp Friedrich Wilhelm Vogt (1789-1861), Dissertation. Giessen, JustusLiebig-Universität, 2008.

35 www.hpgrumpe.de/etschenreutter/lexikon.htm (zugegriffen 2010)

36 Ryff HW: Spiegel der Gesundheit. Frankfurt/M., Christian Egenolffs Erben, 1574. 